\title{
Electronic Transport in Multi-Terminal Graphene Devices with Various Arrangements of Electrodes
}

\author{
S. KROMPIEWSKI* \\ Institute of Molecular Physics, Polish Academy of Sciences, M. Smoluchowskiego 17, 60-179 Poznań, Poland

\begin{abstract}
This study is devoted to the problem of electronic transport in graphene nanodevices in 4-terminal systems with various arrangements of electrodes. The electrodes are attached to square and rectangular graphene nanoflakes with armchair $(a)$ and zigzag $(z)$ edges. Apart from the known case of the $z z z z$-configuration, with all the electrodes coupled to the zigzag fragments of the edges, also the $a a a a$ - and $z a z a$-type cases are considered here. The adopted theoretical approach is based on a tight-binding method combined with the wideband approximation for electrodes, and an effective iterative knitting-type Green's function algorithm.
\end{abstract}

DOI: 10.12693 /APhysPolA.126.194

PACS: 72.80.Vp, 3.63.Rt, 84.32.Dd

\section{Introduction}

Graphene nanostructures have extraordinary physical properties, and are promising materials for numerous practical applications. Widely known graphene's superlatives include exceptional mechanical, thermal, optical and electrical properties. On top of that, graphene is now also known to be of great promise for spintronic applications due to its big spin coherence length, which results from both very weak intrinsic spin-orbit and tiny hyperfine interactions.

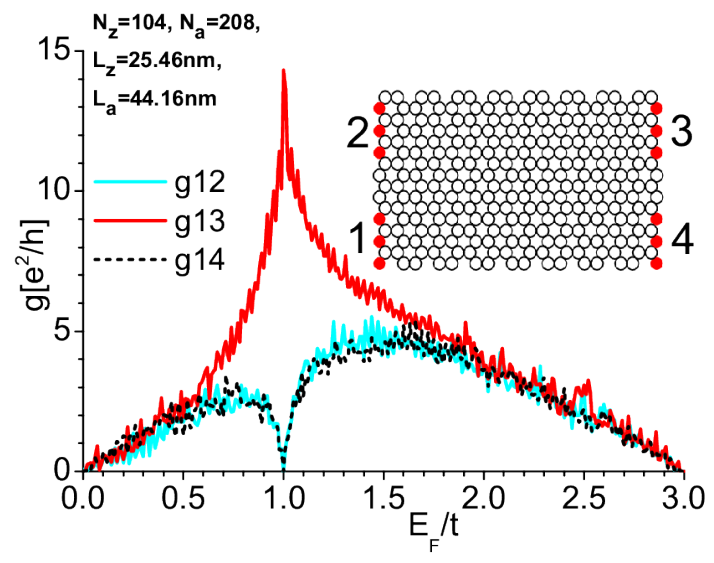

Fig. 1. Graphene nanoribbon with indicated number of outermost edge atoms $N_{z}$ and $N_{a}$, for zigzag and armchair edges respectively. Each of the four electrodes is in contact with 25 edge atoms - red circles (only 3 shown in the schematic inset).

\section{Methodology}

The computations are based on a tight-binding method combined with the Green's function technique. A simple nearest neighbor type Hamiltonian $H_{i j}=t_{i j}+\varepsilon_{i} \delta_{i j}$ is

*e-mail: stefan@ifmpan.poznan.pl used, with $|t|=2.7 \mathrm{eV}$, and an on-site parameter $\varepsilon_{i}$ which is set to zero, except for atoms in contact with the electrodes, for which it is equal to the self-energy. The latter, in the so-called wideband approximation, simplifies to a purely imaginary quantity $\Sigma=-i t_{c}^{2} \operatorname{DOS}\left(E_{F}\right)$, where $t_{c}$ is the hopping integral between the graphene nanoribbon (GNR) and the electrode, and DOS stands for density of states of the electrode. For palladium, Harrison's scaling rule [1] leads to the estimate of $\Sigma \sim-i|t| / 2$. The knitting algorithm is an iterative method to compute Green's function [2], where a single lattice site plays a role of a principle layer used in standard iterative methods $[3,4]$. The knitting method can be successfully used in the multiprobe systems, the main advantage it offers, is no need to invert big matrices. However, a little disadvantage is connected with a formal complexity of the procedure as concerns "sewing", memorizing, updating, and elimination of irrelevant sites. The sewing is based on the duly modified Dyson equation:

$$
\begin{aligned}
G_{A A}^{[A]} & =1 /\left(E-\varepsilon_{A}-\sum_{i, j} t_{A i} G_{i j}^{[A-1]} t_{j A}\right) \\
G_{\alpha A}^{[A]} & =\sum_{i} G_{\alpha i}^{[A-1]} t_{i A} G_{A A}^{[A]}, G_{A \beta}^{[A]}=\sum_{i} G_{A A}^{[A]} t_{A i} G_{i \beta}^{[A-1]} \\
G_{\alpha \beta}^{[A]} & =G_{\alpha \beta}^{[A-1]}+G_{\alpha A}^{[A]} \frac{1}{G_{A A}^{[A]}} G_{A \beta}^{[A]} .
\end{aligned}
$$

Equation (1) adds a new atom $A$ to the system of $A-1$ atoms, and makes it possible to compute new matrix elements of $G$. The last line in Eq. (1) updates all the relevant Green's function elements (see [2] for details). In terms of the reduced Green function matrix, it is straightforward to compute the conductance as $g_{\alpha \beta}=\left(2 e^{2} / h\right) \operatorname{Tr}\left[\Gamma_{\alpha} G \Gamma_{\beta} G^{+}\right]$, with the broadening function $\Gamma_{\alpha}=i\left(\Sigma_{\alpha}-\Sigma_{\alpha}^{+}\right)$, and $\alpha, \beta=1, \ldots, 4$.

The ultimate objective of this study is to show how the electrical conductance of a 4-terminal setup depends on the mutual arrangement of the electrodes. Very small external bias is applied to the contacts in the following manner: $v_{1}=V, v_{2}=v_{3}=v_{4}=0$. As a test case, 
a zzzz-type system (all the electrodes contacted to the zigzag corner edges) is presented in Fig. 1. Similar setup to that was studied earlier in [5] with a different method (no knitting) and another way of modeling of contacts. Nevertheless the results are qualitatively the same, proving that the present approach is correct. Incidentally, as noticed in [5] the sharp features of the conductance spectrum around $E_{F} / t \sim 1$ indicate the tendency of the current to flow along the zigzag path due to the trigonal warping effect. Here a number of other arrangements of electrodes have been considered. It is shown below that the respective electrical performances change quite strongly indeed. This is visualized in Figs. 2 and 3 for the $a a a a$ - and zaza-type arrangements. The former configuration has got the switching functionality, whereas the latter shows that the diagonal propagation direction is the most advantageous for all energies (gate voltages).

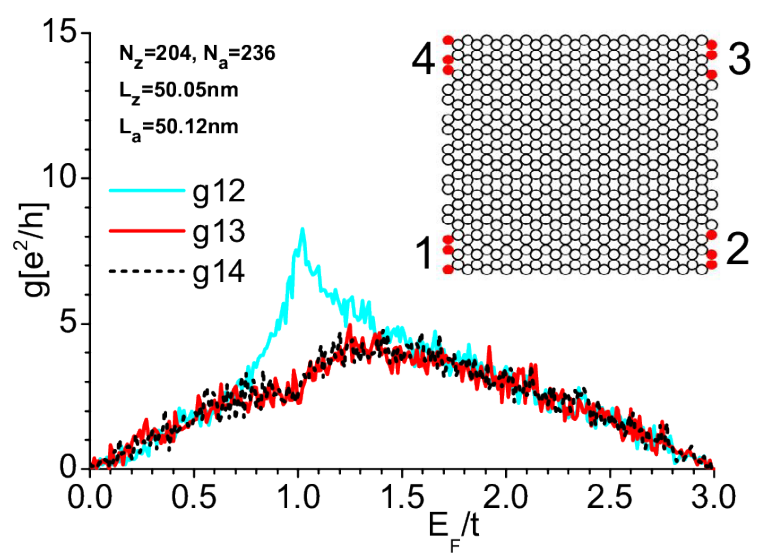

Fig. 2. Conductance of square graphene flakes with the aaaa-type of the electrodes.

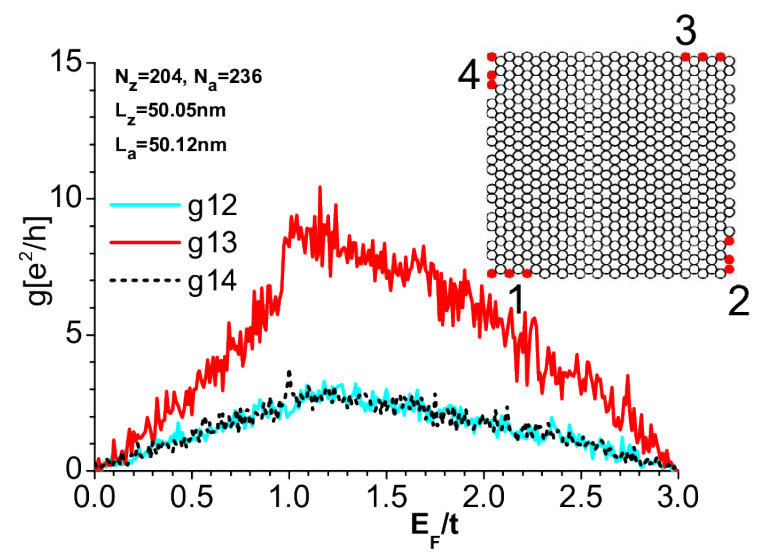

Fig. 3. As Fig. 2 but for the $z a z a$-arrangement.

Finally a system with 4 centrally located electrodes has been also studied. In Fig. 4, all electrodes are ferromagnetic. For the parallel alignment of magnetizations $(\uparrow \uparrow)$ the broadening functions $\Gamma_{\alpha}$-s at the contacts are parameterized as follows $(1.5 ; 1.5 ; 1.5 ; 1.5) t$ and $(05 ; 0.5 ; 0.5$; $0.5) t$ for $\sigma=\uparrow$ and $\downarrow$ spin electrons, respectively. For the $\uparrow \downarrow$-alignment the corresponding numbers are $(1.5 ; 0.5$; $0.5 ; 0.5) t$ and $(0.5 ; 1.5 ; 1.5 ; 1.5) t$. This parametrization corresponds to a $50 \%$ polarization of magnetic electrodes, $\Gamma_{\alpha, \sigma}=\Gamma_{\alpha}(1 \pm p) ; p=0.5$ (cf. [6]). It is readily seen from Fig. 4 that in the ferromagnetic case there is a noticeable spin-valve effect in the longitudinal direction, which reveals itself in the difference in $g_{13}^{\uparrow \uparrow}$ and $g_{13}^{\uparrow \downarrow}$, however the effect in the transversal direction is much weaker.

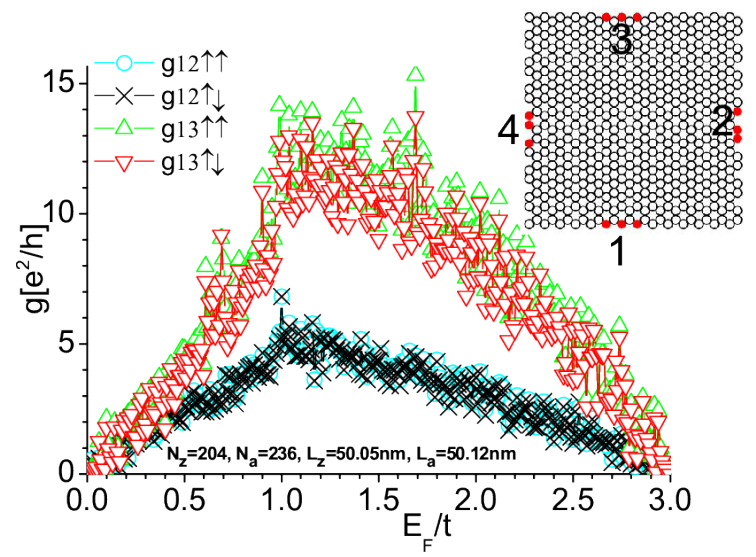

Fig. 4. Conductance of square graphene flakes with centrally located electrodes. The electrodes are ferromagnetic. In the $\uparrow \uparrow$-configuration all the magnetizations are parallel to each other, whereas for the $\uparrow \downarrow-$ configuration the electrode 1 is antiparallel magnetized to the others. There is a visible spin-valve effect for the longitudinal direction (13).

\section{Conclusions}

Summarizing, a simple implementation of the knitting algorithm to multi-probe graphene-based systems has been demonstrated. Mutual arrangements of the electrodes determine, to much extent, conductance of particular channels between the contacts. The results provide additional insight into electronic transport properties of four-terminal setups, and show when the gate-controlled switching functionality can be realized and how to select optimum conduction paths. It has also been shown that, if ferromagnetic contacts are used, the spin-valve effect in the longitudinal direction is stronger than that in the transverse direction.

\section{Acknowledgments}

This work was supported by the Polish Ministry of Science and Higher Education as a research project No. N N202 199239 for 2010-2013.

\section{References}

[1] W.A. Harrison, Electronic structure and the properties of solids, Ed. W.H. Freeman, San Francisco, 1980.

[2] K. Kazymyrenko, X. Waintal, Phys Rev. B 77, 115119 (2008).

[3] S. Krompiewski, Phys. Rev. B 80, 075433 (2009).

[4] S. Krompiewski, Nanotechnology 23, 135203 (2012).

[5] K. Sääskilahti, A. Harju, P. Pasanen, Appl. Phys. Lett. 95, 092104 (2009).

[6] I. Weymann, J. Barnaś, S. Krompiewski, Phys. Rev. B 85, 205306 (2012). 